\title{
TWO SUBROUTINES FOR CALCULATING LATTICE SUMS AND THE DISTORTION FIELD DUE TO A POINT FORCE IN HEXAGONAL SYSTEMS
}

\author{
W. MAYSENHÖLDER \\ Max-Planck-Institut (MPI) für Metallforschung, Institut für Physik, Stuttgart, Fed. Rep. Germany
}

Received 4 May 1979; in revised form 22 May 1981

\section{PROGRAM SUMMARY}

Title of program: HEXALAT

Catalogue number: QCKQ

Program obtainable from: CPC Program Library, Queen's University of Belfast, $N$. Ireland (see application form in this issue)

Computer: Honeywell Bull 66/80 DPS; Installation: MPI Stuttgart

Operating system: GCOS

Programming language used: FORTRAN

High speed store required: 23 kwords

No. of bits in a word: 36

Overlay structure: none

No. of magnetic tapes required: none

Other peripherals used: card reader, line printer

No. of cards in combined program and test deck: 445

Card punching code: IBM 29

Keywords: solid state physics, hexagonal systems, elasticity theory, continuum Green's function, lattice sums, location and diffusion of interstitials

Nature of physical problem In solid state theory the calculation of lattice sums in a perfect as well as in a distorted lattice is frequently required. HEXALAT contains two subroutines which can be used for calculations of this kind in the case of hexagonal systems. The subroutine ARRANGE generates the coordinates of a roughly spherical arrangement of atoms occupying the regular sites of an hcp structure. The coordinates are all stored in one array and can be used for calculating lattice sums. The subroutine DISPL calculates the displacement of an atom due to a double force tensor centered at the origin of the coordinate system in the elastic approximation. As an example, HEXALAT calculates the potential energy of an interstitial at an octahedral site both in the undistorted and in the distorted hexagonal lattice. The interaction potential between the interstitial and one atom is assumed to be given. Calculations of this kind are essential for the determination of the most stable sites for an interstitial and for the investigation of the diffusive behaviour.

\section{Method of solution}

The procedure used by ARRANGE is elementary and described in detail in the long write-up. DISPL uses the analy tic expressions for the derivatives of the continuum Green's function. The parameters needed by DISPL depend on the elastic constants and are supplied by the subroutine PARA.

Restrictions on the complexity of the problem HEXALAT is written in single precision. DISPL is restricted to the evaluation of displacements due to a diagonal double force tensor $P$ with $P_{11}=P_{22}$ (which holds for point defects at usual interstitial sites).

Typical running time

The execution time depends on the number of atoms in the arrangement. The test run (180 atoms) on the Honeywell Bull 66/80 DPS took $2.1 \mathrm{~s}$ for compilation and $2.0 \mathrm{~s}$ for execution. 


\section{LONG WRITE-UP}

\section{Introduction}

The subroutines ARRANGE and DISPL contained in HEXALAT have been developed for application of solid state theory to hexagonal crystals, in particular to the hexagonal closed packed (hcp) structures.

ARRANGE generates the coordinates of a roughly spherical arrangement of atoms which occupy the regular sites of an hcp structure, and thus provides a means for calculating lattice sums very easily. (The method of summation over shells of atoms, which is often applied to cubic crystals, is not applicable to hcp crystals.) Another frequent problem, the distortion of the lattice due to a point defect, is treated by the routine DISPL. The displacement of an atom is calculated in the elastic approximation using the analytic expressions of the derivatives of the continuum Green's function [1]. In many cases the accuracy of the elastic approximation is sufficient [2] and the extensive calculations necessary for the determination of the lattice Green's function can be avoided *.

The use of ARRANGE and DISPL is demonstrated in the present test program, which calculates the potential energy of an interstitial at an octahedral site both in the undistorted and in the distorted lattice. However, because for the test run the interaction potential between the interstitial and one atom is simply replaced by a "test function" simulating a screened Coulomb potential, the energies and displacements shown in the test run output do not refer to a particular interstitial. Both routines will be described in detail in sections 2 and 3.

\section{ARRANGE}

ARRANGE generates the coordinates of a roughly spherical arrangement of atoms occupying the regular sites of an hcp structure. The approximate radius RMAX of this arrangement has to be set by the user.

\footnotetext{
* A program for the numerical determination of the elastic Green's function and its derivatives for cubic crystals has been developed by R.K. Leutz and R. Bauer, Comput. Phys. Commun. 11 (1976) 339.
}

The coordinates are stored in the array RAT(NRAT, 3). NRAT must be greater than or equal to NMAX, the total number of atoms in the arrangement. If not, an error message is printed and the program is stopped. In the test run version NRAT is set to 2000, which should be sufficient for RMAX $<6 a$ ( $a, c$ are the lattice constants). If RMAX $>6 a$, NRAT can be estimated by $10(\mathrm{RMAX})^{3} / a^{2} c$.

The lattice vectors $a, b, c$ and the vector $t_{\mathrm{B}}$ to the second atom of the basis are defined by

$$
\begin{aligned}
& a=\left[\begin{array}{l}
-\frac{a}{2} \\
-\frac{1}{2} \sqrt{3} a \\
0
\end{array}\right], \quad b=\left(\begin{array}{l}
a \\
0 \\
0
\end{array}\right), \\
& c=\left(\begin{array}{l}
0 \\
0 \\
c
\end{array}\right), \quad t_{\mathrm{B}}=\left(\begin{array}{l}
0 \\
-\frac{1}{3} \sqrt{3} a \\
\frac{c}{2}
\end{array}\right] .
\end{aligned}
$$

$\boldsymbol{a}$ and $\boldsymbol{b}$ define a basal plane, $\boldsymbol{c}$ is parallel to the hexagonal axis.

The routine proceeds plane by plane and starts with the atom at $g_{1}=(0,0,-n c)$, where $n$ is the greatest integer less than $\mathrm{RMAX} / c$. The position of the second atom in this plane is obtained by $g_{2}=$ $g_{1}+a$. Similarly, $g_{3}=g_{2}+b, g_{4}=g_{3}+(a+b)$. The fifth atom is reached by $g_{5}=g_{4}-(a+2 b)$ and constitutes the beginning of a new round, which leads by means of suitable combinations of $\boldsymbol{a}$ and $\boldsymbol{b}$ counterclockwise around the first four atoms and yields the coordinates of ten more atoms. The third round is introduced by $g_{15}=g_{14}-(a+2 b)$ and contains 16 atoms, etc. However, if the starting point of a round has a distance from the origin greater than RMAX, the coordinates of that atom are not stored, and the algorithm is stopped and restarted in the basal plane above. In this way one obtains the coordinates of a roughly spherical hexagonal point lattice which are stored in the odd elements of the array RAT, i.e. $g_{1}=$ $\{\operatorname{RAT}(1, \mathrm{I})\}, \boldsymbol{g}_{2}=\{\operatorname{RAT}(3, \mathrm{I})\}$, etc. $(\mathrm{I}=1,2,3)$. The hexagonal closed packed structure consists of two hexagonal Bravais lattices which are displaced by the vector $t_{\mathrm{B}}$. The coordinates of the second sublattice, 
which are stored in the even elements of RAT, are simply obtained by adding $t_{\mathrm{B}}$ to the coordinates of the respective preceding (odd) elements.

The hcp arrangement constructed this way is still roughly spherical (provided RMAX is not too small). It should be noted that strictly speaking it does not possess any of the symmetries of an infinite hep structure (not even the threefold symmetry). However, the size of the arrangement has to be chosen so large anyway that the influence of the surface atoms on the central region can be neglected. Therefore, the lack of symmetry does not matter in practical applications.

\section{DISPL}

Generally, the displacements $s$ of a mass element at location $\boldsymbol{R}$ of an elastic medium due to a force density $f(r)$, which is non-zero only for "relatively small $\boldsymbol{r}$ ", can be obtained in terms of the derivatives of the elastic (continuum) Green's function $S$ and the double force tensor $\boldsymbol{P}[3]$.

$s_{i}(R)=-\sum_{j k} P_{j k} \frac{\partial}{\partial x_{j}} S_{i k}(R)$

with

$P_{j k}=\int \mathrm{d}^{3} r^{\prime} x_{j}^{\prime} f_{k}\left(r^{\prime}\right)$.

Eq. (1) holds in lattice theory if the lattice Green's function is used instead of the continuum one and the double force tensor is given by

$P_{j k}=\sum_{n} x_{j}^{(n)} F_{k}^{(n)}$.

The $x_{j}^{(n)}$ are the Cartesian components of the location $R^{(n)}$ of the atom $n$, the $F_{k}^{(n)}$ are the components of the force $F^{(n)}$ exerted on that atom (e.g. by the point defect). It has been shown $[2,3]$ that even for small distances from the defect the continuum Green's function is a rather good approximation to the lattice Green's function. Thus one can take advantage of the fact that an analytic expression for $S$ exists for hexagonal systems [1].

In most cases of interest one is concerned with a diagonal double force tensor with $P_{11}=P_{22}$. (This applies in particular to defects at octahedral, tetrahedral, trigonal and hexahedral interstitial sites.) Then only the following derivatives of $S$ are needed which have been evaluated from the expression given in ref. [1].

$\frac{\partial}{\partial x} S_{11}+\frac{\partial}{\partial y} S_{12}=\sum_{l=1}^{3} t_{l}\left(A_{l} a_{l}-B_{l}\right) a_{l} x$

$\frac{\partial}{\partial z} S_{13}=\sum_{l=1}^{3} t_{l} C_{l} a_{l} x$

$\frac{\partial}{\partial x} S_{21}+\frac{\partial}{\partial y} S_{22}=\sum_{l=1}^{3} t_{l}\left(A_{l} a_{l}-B_{l}\right) a_{l} y$

$\frac{\partial}{\partial z} S_{23}=\sum_{l=1}^{3} t_{l} C_{l} a_{l} y$

$\frac{\partial}{\partial x} S_{31}+\frac{\partial}{\partial y} S_{32}=\sum_{l=1}^{3}-t_{l} C_{l} a_{l} z$

$\frac{\partial}{\partial z} S_{33}=\sum_{l=1}^{3}-t_{l} D_{l} z$

with

$t_{l}=\left(a_{l}\left(x^{2}+y^{2}\right)+z^{2}\right)^{-3 / 2}$.

The parameters $A(l), B(l), C(l), D(l), a(l)$ depend on the elastic constants $C_{i j}$ and are evaluated by the subroutine PARA, which has to be called once by the main program before the first call of DISPL. The formulae read

$A_{l}=\left[\left(C_{66}-C_{11}\right)\left(C_{33}-a_{l} C_{44}\right)+\left(C_{13}+C_{44}\right)^{2}\right] \frac{1}{E_{l}}$,

$B_{l}=\left[\left(C_{44}-a_{l} C_{11}\right)\left(C_{33}-a_{l} C_{44}\right)+a_{l}\left(C_{13}+C_{44}\right)^{2}\right] \frac{1}{E_{l}}$,

$C_{l}=\left(C_{13}+C_{44}\right)\left(C_{44}-a_{l} C_{66}\right) \frac{1}{E_{l}}$,

$D_{l}=\left(C_{44}-a_{l} C_{11}\right)\left(C_{44}-a_{l} C_{66}\right) \frac{1}{E_{l}}$,

$E_{1}=-4 \pi\left(a_{1}-a_{2}\right)\left(a_{3}-a_{1}\right) K$

and cycl.,

$K=C_{11} C_{44} C_{66}$.

$a_{1}=C_{44} / C_{66} ; a_{2}, a_{3}$ are the roots of the equation

$C_{11} C_{44} a^{2}+\left(C_{13}^{2}+2 C_{13} C_{44}-C_{11} C_{33}\right) a+C_{33} C_{44}=0$. 
(The factor $-K$ is missing in ref. [1]! Remember that $C_{66}=\frac{1}{2}\left(C_{11}-C_{12}\right)$. The parameters may become complex.)

The components of the double force tensor must be supplied by the user. They may be calculated from the derivative of the interaction potential $U(r)$ between the interstitial and one atom. For the test run only nearest neighbours to an octahedral interstitial (with distance $r_{N N}$ ) have been taken into account:

$$
\begin{aligned}
& P_{11}=-\left.\frac{\partial U(r)}{\partial r}\right|_{r_{N N}} \frac{a^{2}}{r_{N N}}, \\
& P_{33}=-\left.\frac{\partial U(r)}{\partial r}\right|_{r_{N N}} \frac{3 c^{2}}{8 r_{N N}} .
\end{aligned}
$$

\section{A list of the subroutines in the deck}

The program for the test run consists of a main program and the following subroutines:
1) ARRANGE
2) DISPL
3) PARA

4) SUM

5) DIST

6) EQUAL

7) RITE

described in section 2 , described in section 3 , evaluates the parameter needed by DISPL, calculates the sum of two 3-dimensional vectors, is a FUNCTION-subprogram which calculates the distance between two points, puts a 3-dimensional vector into the (NRAT, 3)-matrix RAT, performs the printing of the coordinates and the displacements of those atoms which have a distance less than ROUT from the origin.

\section{Calling sequences for the subroutines}

1) The calling sequence for ARRANGE is

\section{CALL ARRANGE(RAT,RMAX,NMAX,NRAT,AA,CC)} where

RAT is a (NRAT, 3)-matrix containing the coordinates of the atoms (output of ARRANGE),
RMAX is the approximate radius of the arrangement (input),

NMAX is the total number of atoms in the arrangement (output),

NRAT specifies the dimension of RAT (input), $\mathrm{AA}, \mathrm{CC}$ are the lattice constants $a$ and $c$ (input).

2) The calling sequence for DISPL is

CALL DISPL(R,DR,P11,P33,A,B,C,D,SA)

where

$R$ is a 3-dimensional vector containing the coordinates of the atom to be displaced (input),

DR is a 3-dimensional vector representing the displacement (output),

P11, P33 are the diagonal components of the double force tensor (input),

A, B, C, are 3-dimensional complex vectors repreD, SA senting the parameters which have to be supplied by PARA (input).

3) The calling sequence for PARA is

CALL PARA(C11,C13,C33,C44,C66,A,B,C,D,SA) where

$\mathrm{C} 11, \mathrm{C} 13, \mathrm{C} 33$, are the elastic constants (input), $\mathrm{C} 44, \mathrm{C} 66$

A, B, C, D, SA are 3-dimensional complex vectors representing the parameters needed by DISPL (output).

PARA has to be called once from the main program before the first call of DISPL.

4) The calling sequence for SUM is

CALL SUM(A, B, AB)

where

$A, B, A B$ are 3-dimensional vectors obeying the relation $\mathbf{A}+\mathbf{B}=\mathbf{A B}$.

SUM is called from ARRANGE.

5) The calling sequence for DIST is

$\mathrm{D}=\operatorname{DIST}(\mathrm{A}, \mathrm{B})$

where A, B are 3-dimensional vectors. The function value $D$ is the modulus of their difference.

DIST is called from ARRANGE. 
6) The calling sequence for EQUAL is

\section{CALL EQUAL(RAT, NRAT, G, N)}

where

RAT, NRAT
G
N

are described in 1), is a 3-dimensional vector, is an integer specifying the elements of RAT which have to be set equal to the components of $\mathrm{G}$.

EQUAL is called from ARRANGE.

7) The calling sequence for RITE is

\section{CALL RITE(RAT,DRAT,NRAT,NMAX,ROUT,J)}

where

$\begin{array}{ll}\text { RAT, NRAT, NMAX } & \text { are described in 1), } \\ \text { is a (NRAT; 3)-matrix con- } \\ \text { taining the displacements, } \\ \text { specifies the radius of a } \\ \text { sphere. The coordinates (and } \\ \text { eventually the displacements) } \\ \text { of all atoms within this sphere } \\ \text { are printed, } \\ =1: \text { the displacements are not } \\ \text { printed, } \\ \text { J } 2: \text { the displacements are } \\ \text { printed. }\end{array}$

RITE is called from the main program.

\section{Input required}

The main program calling ARRANGE has to supply the lattice constants $\mathrm{AA}$ and $\mathrm{CC}$ and the approximate radius RMAX of the arrangement. RMAX in turn determines the dimension NRAT of the array RAT, which also has to be specified by the user (see section 2). A further parameter ROUT has to be fixed if printing of coordinates (and displacements) by RITE is desired. For calculating displacements the main program has to deliver the elastic constants $C_{i j}$ for the subroutine PARA and the components P11 and P33 of the double force tensor as well as the location $\mathbf{R}$ of the atom to be displaced for the subroutine DISPL. The present main program sets these input variables in DATA statements.

\section{Test run}

The present main program shows an example of the use of ARRANGE and DISPL. It calculates the potential energy of an interstitial at an octahedral site $\left(r_{\text {octa }}=\left(\frac{1}{2} a,-(\sqrt{3} / 6) a, c / 4\right)\right)$ both in an undistorted and in a distorted hcp lattice. The additional input required for this purpose is the interaction potential $U(r)$ between the interstitial and one atom. In order to supply an easily reproducible test run we simply set $U(r)=(1 / r) \exp (-r)$ which describes some repulsive interaction. The potential energy is determined by

$E\left(r_{\text {octa }}\right)=\sum_{n} U\left(\left|r_{\text {octa }}-R^{(n)}\right|\right)$.

The test run output shows the coordinates of those atoms, which lie within a sphere of radius ROUT $=7$ around $r_{\text {octa }}$, in a Cartesian system with the origin at the octahedral site. (The origin of the system used by ARRANGE has been shifted by $r_{\text {octa }}$.) The label $\mathrm{N}$ specifies the position of the coordinates of an atom within the array RAT. The potential energy of the interstitial at $\boldsymbol{r}_{\text {octa }}$ is denoted by $E(1)$. As a control the potential energy $E(2)$ at an (equivalent) adjacent octahedral site is also calculated. The agreement of $E(1)$ and $E(2)$ indicates that RMAX is large enough to achieve an accuracy of four digits with the "test interaction potential" $(1 / r) X$ $\exp (-r)$. After the complex parameters from PARA the double force tensor is printed, which has been derived from $U(r)$ according to eq. (2) of section 3 taking into account nearest neighbours only. Then each atom of the arrangement is displaced via DISPL and the potential energy $\mathrm{E}(1)$ calculated again.

The lattice parameters and elastic constants are those of beryllium at $80 \mathrm{~K}$ (in atomic units). It is recommended to use atomic units $(\hbar=m=e=1)$ in order to avoid exponential underflow or overflow which otherwise may occur depending on the computer used.

\section{References}

[1] E. Kröner, Z. Phys. 136 (1953) 402.

[2] H.R. Schober, M. Mostoller and P.H. Dederichs, Phys. Stat. Sol. (b) 64 (1974) 173.

[3] G. Leibfried and N. Breuer, Point defects in metals I (Springer Tracts in Modern Physics 81, Berlin, 1978). 


\section{TEST RUN OUTPUT}

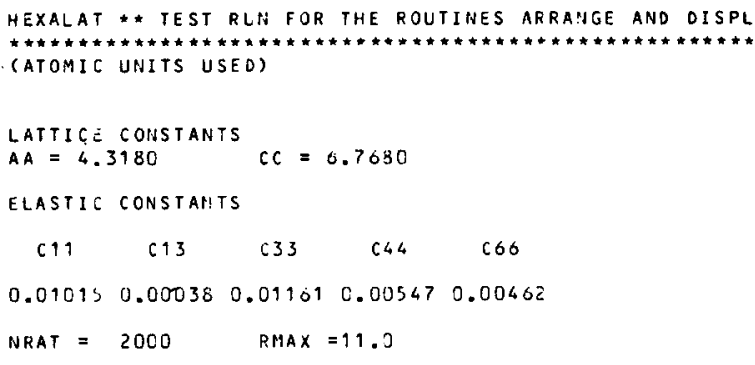

NUMBEP CF ATOMS IHi IHE ARRANGEMENT IMAX $=190$

COORDIIATES RELATIVE TO OCTAIIEDHAL SITE

$$
\text { LOCATIUH OF ATONS }
$$

\begin{tabular}{|c|c|c|c|}
\hline N & $x$ & 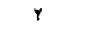 & 2 \\
\hline 2 & -2.1550 & -1.2465 & -5.0760 \\
\hline 8 & 2.1540 & -1.2465 & -5.0760 \\
\hline 23 & -0.0000 & 2.4930 & -5.0760 \\
\hline 61 & -2.1590 & 1.2465 & -1.6920 \\
\hline 62 & -2.1590 & -1.2405 & 9.6920 \\
\hline 63 & -4.3180 & -2.4930 & -1.6920 \\
\hline 64 & $-4.31<0$ & -4.7565 & 1.6920 \\
\hline$\epsilon 5$ & 0 & -2.4930 & -1.6920 \\
\hline 66 & 0. & -4.9800 & 1.0920 \\
\hline $6 ?$ & 2.1590 & 1.2405 & -1.0920 \\
\hline 65 & 2.1540 & -1.2405 & 1.0920 \\
\hline 69 & -4.3180 & 4.9860 & -1.5920 \\
\hline$? 0$ & -4.3120 & 2.4930 & 1.6920 \\
\hline 71 & -6.4770 & 1.2405 & -1.6920 \\
\hline 72 & -6.4770 & -1.2465 & 1.6920 \\
\hline 77 & -2.1590 & -6.2325 & -1.6920 \\
\hline 79 & 2.1590 & -6.2325 & -1.6920 \\
\hline 81 & 4.3180 & -2.4430 & -1.6920 \\
\hline 82 & 4. 3180 & -4.9660 & 1.6920 \\
\hline 83 & 0.4 .770 & 1.2465 & -1.3920 \\
\hline 84 & 6.4770 & -1.2465 & 1.0920 \\
\hline 85 & 4.3180 & 4.9863 & -1.6920 \\
\hline 86 & 4.3180 & 2.4930 & 1.6920 \\
\hline 87 & -0.0000 & 4.9860 & -1.6920 \\
\hline 88 & -0.0000 & 2.4935 & 1.6920 \\
\hline 18 & 2.1590 & 0.2325 & 1.6920 \\
\hline 20 & -2.1590 & 0.2325 & 1.6920 \\
\hline 21 & -2.1590 & 1.2465 & 5.0760 \\
\hline 25 & 0. & -2.4933 & 5.0760 \\
\hline 27 & 2.1540 & 1.2465 & 5.0760 \\
\hline
\end{tabular}

(THE O JTPUT IS LIPITED TO ATOMS WITHIH A SPHERE OF RADIUS ROUT $=7.0$ AROUND THE ORIGI!.

N DENOTES THE FIRST INDEX OF ARRAY RAT.

POTENTIAL ENERGY OF AN INTERSTITIAL AT OCTAHEDRAL SITES $E(1)=0.1108 \quad E(2)=0.110 \varepsilon$ 


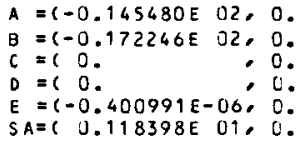

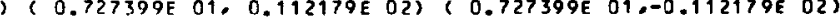

260.

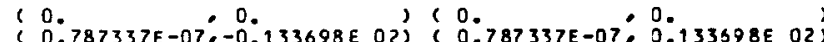

)$(0$.

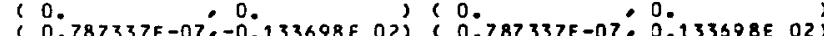
$(-0.727399 \mathrm{E}$ 01, $0.112179 \mathrm{E}$ 02) $(-0.727399 \mathrm{E} \quad 01,-0.112179 \mathrm{E}$ O2) )$(-0.727399 E$ OI, 0.112179 E 02$)$ ( $-0.727399 \mathrm{E} 01,-0.112179 \mathrm{E} 02)$ \}$\left(\begin{array}{l}0.633883 E-06,0.326427 E-06)(0.633883 E-06,-0.326427 E-06) \\ (0.102250 E 01,0.313572 E 00)(0.102250 E 01,-3.313572 E \text { OO }\end{array}\right.$

DOUBLE FORCE TENSOR

P11 $=3.1344 \quad$ P33 $=0.1238$

COORDIMATES AFTER OISTORTION

LOCATION OF ATOMS

\begin{tabular}{|c|c|c|c|}
\hline$N$ & $x$ & $Y$ & $z$ \\
\hline $\begin{array}{l}2 \\
8\end{array}$ & -2.1710 & -1.2535 & -5.0969 \\
\hline 8 & 2.1710 & -1.2535 & -5.0969 \\
\hline 28 & -0.0000 & 2.5069 & -5.0969 \\
\hline 61 & -2.2436 & 1.2953 & -1.7457 \\
\hline 62 & $-2.24 \geq 6$ & -1.2953 & 1.7457 \\
\hline 63 & -4.3490 & -2.5113 & -1.7024 \\
\hline 64 & -4.3326 & -5.0029 & 1.6968 \\
\hline 65 & 0 & -2.5907 & -1.7457 \\
\hline 66 & 0 & -5.0225 & 1.7024 \\
\hline 67 & 2.2430 & 1.2953 & -1.7457 \\
\hline 63 & 2.2436 & -1.2953 & 1.7457 \\
\hline 69 & -4.3326 & 5.3329 & -1.6968 \\
\hline 75 & -4.3496 & 2.5193 & 1.7024 \\
\hline 71 & -6.4989 & 1.2507 & -9.6968 \\
\hline 72 & $-6.49 \varepsilon ?$ & -1.2507 & 1.6988 \\
\hline 77 & -2.1663 & -0.2536 & -1.6968 \\
\hline 79 & 2.1663 & -0.2536 & -1.6968 \\
\hline 89 & 4.3496 & -2.5113 & -1.7024 \\
\hline 82 & 4.3320 & -5.0029 & 1.6968 \\
\hline 83 & 6.4989 & 1.2507 & -1.6968 \\
\hline 84 & 6.4989 & -1.2507 & 1.6968 \\
\hline 35 & 4.3326 & 5.0029 & -1.6968 \\
\hline 85 & 4.3496 & 2.5113 & 1.7024 \\
\hline 87 & -0.0000 & 5.0225 & -1.7024 \\
\hline 83 & -0.0000 & 2.5907 & 1.7457 \\
\hline 118 & 2.1663 & 6.2536 & 1.6968 \\
\hline 123 & -2.1603 & 0.2536 & 1.6968 \\
\hline 121 & -2.1710 & 1.2535 & 5.0969 \\
\hline 125 & 0. & -2.5069 & 5.0969 \\
\hline 127 & 2.1710 & 1.2535 & 5.0969 \\
\hline
\end{tabular}

OISPLACEMENTS.

DX DY DZ

$\begin{array}{lll}-0.0120 & -0.0070 & -0.0209\end{array}$

$0.0120-0.0070-0.0209$

$\begin{array}{lll}-0.0000 & 0.0139 & -0.0209\end{array}$

$\begin{array}{lll}-0.0846 & 0.0488 & -0.0537\end{array}$

$\begin{array}{lll}-0.0846 & -0.0488 & 0.0537\end{array}$

$\begin{array}{lll}-0.0316 & -0.0183-0.0104\end{array}$

$\begin{array}{lll}-0.0146 & -0.0169 & 0.0048\end{array}$

$0 . \quad-0.0977-0.0537$

$\begin{array}{lll}0.0 & -0.0365 & 0.0104\end{array}$

$\begin{array}{lll}0.0846 & 0.0488 & -0.0537\end{array}$

$\begin{array}{lll}0.0846 & -0.0488 & 0.0537\end{array}$

$\begin{array}{lll}-0.0146 & 0.0169 & -0.0048\end{array}$

$\begin{array}{rrr}-0.0316 & 0.0183 & 0.0104 \\ -0.0219 & 0.0042 & -0.0048\end{array}$

$\begin{array}{rrr}-0.0219 & 0.0042 & -0.0048 \\ -0.0219 & -0.0042 & 0.0048\end{array}$

$\begin{array}{rrr}-0.0219 & -0.0042 & 0.0048 \\ -0.0073 & -0.0211 & -0.0048\end{array}$

$0.0073 \quad-0.0211-0.0048$

$0.0316 \quad-0.0183-0.0104$

$0.0146 \quad-0.0169 \quad 0.0048$

$\begin{array}{lll}0.0219 & 0.0042 & -0.0048\end{array}$

$\begin{array}{lll}0.0219 & -0.0042 & 0.0048\end{array}$

$\begin{array}{lll}0.0146 & 0.0169 & -0.0048\end{array}$

$\begin{array}{rrr}0.0146 & 0.0169 & -0.0048 \\ 0.0316 & 0.0183 & 0.0104\end{array}$

$\begin{array}{rrr}-0.0000 & 0.0365 & -0.0104\end{array}$

$\begin{array}{rrr}-0.0000 & 0.0365 & -0.0104 \\ -0.0000 & 0.0977 & 0.0537\end{array}$

$\begin{array}{rrr}-0.0000 & 0.0977 & 0.0537 \\ -0.0073 & 0.0211 & 0.0048\end{array}$

$\begin{array}{llll}-0.0073 & 0.0211 & 0.0048\end{array}$

$\begin{array}{llll}-0.0120 & 0.0070 & 0.0209\end{array}$

$0 . \quad-0.0139 \quad 0.0209$

$\begin{array}{lll}0.0120 & 0.0070 & 0.0209\end{array}$

(THE OJTPUT IS LIMITED TO ATOME WITHIN A SPHERE

OF RADIUS ROUT $=7.0$ AROUND THE ORIGIN.

N DENOTES THE FIRST INDEX OF ARRAY RAT.

ENERGY E(1) AFTER DISTORTION 0.0970 